\title{
Unsteady flow regimes in arrow-shaped micro-mixers with different tilting angles
}

Cite as: Phys. Fluids 33, 012008 (2021); https://doi.org/10.1063/5.0033765

Submitted: 20 October 2020 . Accepted: 14 December 2020 . Published Online: 14 January 2021

(D) A. Mariotti, (D) C. Galletti, (D) E. Brunazzi, and (D) M. V. Salvetti

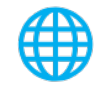

\section{Physics of Fluids GALLERY OF GOVERS}




\title{
Unsteady flow regimes in arrow-shaped micro-mixers with different tilting angles
}

\author{
Cite as: Phys. Fluids 33, 012008 (2021); doi: 10.1063/5.0033765 \\ Submitted: 20 October 2020 - Accepted: 14 December 2020 • \\ Published Online: 14 January 2021
}

\section{A. Mariotti, ${ }^{\text {a) }}$ (D) C. Galletti, ${ }^{\text {b) }}$ (D) E. Brunazzi, ${ }^{\text {b) }}$ (D) and M. V. Salvetti}

\author{
AFFILIATIONS \\ Dipartimento di Ingegneria Civile e Industriale, Università di Pisa, Largo L. Lazzarino 2, 56122 Pisa, Italy \\ a) Electronic mail: alessandro.mariotti@unipi.it \\ b) Authors to whom correspondence should be addressed: chiara.galletti@unipi.it and elisabetta.brunazzi@unipi.it \\ ${ }^{c}$ Electronic mail: mv.salvetti@ing.unipi.it
}

\begin{abstract}
Two arrow-shaped micro-mixers, obtained from the classical T-shaped geometry by tilting downward the inlet channels, are considered herein. The two configurations, having different tilting angle values, have been chosen since they show significantly different flow topologies and mixing performances at low Reynolds numbers. In the present paper, we use both experimental flow visualizations and direct numerical simulations to shed light on the mixing behavior of the two configurations for larger Reynolds numbers, for which the mixers present unsteady periodic flows, although in laminar flow conditions. The tilting angle influences the flow dynamics also in the unsteady regimes and has a significant impact on mixing. The configuration characterized by the lower tilting angle, i.e., $\alpha=10^{\circ}$, ensures a better global mixing performance than the one with the larger angle, i.e., $\alpha=20^{\circ}$.
\end{abstract}

Published under license by AIP Publishing. https://doi.org/10.1063/5.0033765

\section{INTRODUCTION}

Micro-mixers and micro-reactors are constituted by channels of width $\leq 1 \mathrm{~mm}$, in which two fluid streams are fed through inlet branches and mix and possibly react in the outlet channel. They have been widely proposed in the last decade for several industrial applications because they ensure an unprecedented control over the reaction conditions, thanks to the continuous operation and the strong heat transfer due to the large surface-to-volume ratio. The latter assures dealing safely with exothermic reactions, while the continuous flow avoids also the accumulation of toxic and reactive species, thus widening the spectra of reactions and conditions that can be run. Hence, micro-fluidic devices may provide significant environmental, safety, and economic benefits, allowing the intensification of many fine-chemistry and pharmaceutical processes.

Efficient mixing is fundamental to obtain high reaction yields. ${ }^{4}$ Many active or passive methods have been proposed to promote mixing. ${ }^{5-9}$ The active ones need ultrasounds, electric fields, pressure pulses, and so on as an extra energy input, while the passive ones want to break the symmetry of the flow through a favorable design of the geometry of the mixer without any external energy input.

The T-shaped geometry-in which the axis of the two inlet channels is perpendicular to that of the main mixing channel-is the simplest and most studied configuration of micro-mixers. Despite the very simple geometry and the laminar flow conditions, significantly different flow regimes occur when increasing the Reynolds number. The characterization and eventually the control of the occurrence of these regimes are crucial because they can lead to largely different degrees of mixing. The most widely studied regime is the engulfment one because its onset corresponds to the first significant increase in mixing in the device at low Reynolds numbers. Many previous studies were aimed at predicting the critical Reynolds number, $R e$, at which the onset of the engulfment regime and the related increase in mixing occur, and at investigating how it depends on different geometrical and operational parameters. Some investigations, for instance, studied the sensitivity to the aspect ratio of the channels, ${ }^{10-13}$ to the operating conditions, ${ }^{14-16}$ and to the fluid properties, ${ }^{11,17-20}$ while others proposed modifications of the junction shape were aimed to anticipate the onset of the engulfment regime at lower $R e^{21-25}$ 
Among the geometry modifications proposed to promote the engulfment regime, Siconolfi et al. ${ }^{24}$ showed that the downward tilting of the inlet channels (arrow-shaped micro-mixers) makes the flow more sensitive to the instability, leading to the engulfment regime, and hence, the onset of this regime takes place at $R e$ significantly lower than for T-shaped mixers. The opposite occurs for Y-shaped micro-mixers, which are thus less interesting for mixing promotion purposes. The anticipation of the engulfment onset in arrow-shaped mixers is confirmed by the simulations in the work of You et al. ${ }^{22}$ On the other hand, in the numerical simulations of Galletti et al., ${ }^{26}$ a non-monotonic behavior of the degree of mixing with $R e$ was found in arrow-shaped mixers within the engulfment regime, which is conversely not observed for T-mixers. In a recently published paper, ${ }^{27}$ a systematic investigation on the steady regimes occurring in arrow-shaped micro-mixers fed by liquids and with varying the tilting angle $\alpha$ was carried out. Experimental flow visualizations and numerical simulations were used together to study mixers having square inlet sections and a rectangular 2:1 outlet. The anticipation of the engulfment regime onset was found to become more pronounced by increasing the tilting angle $\alpha$. However, confirming the clues in the work of Galletti et al., ${ }^{26}$ a significant drop of mixing was observed within the steady engulfment regime for $\alpha$ larger than some critical value (between $15^{\circ}$ and $20^{\circ}$ for the configuration investigated in the work of Mariotti et al. ${ }^{27}$ ). This is not present for T-mixers and low tilting angles. The mixing reduction is due to a significant change in the topology of the flow, which becomes characterized by a single vortical structure in the center of the mixing channel instead of the two co-rotating ones, present in T-mixers in the engulfment regime. This vortical structure promotes mixing in a less-efficient way compared to the classical engulfment pattern, and the mixing degree is indeed lower than in T-mixers at the same Re. By further increasing $R e$, the mixing degree increases again-due to the increased size and strength of the vortical structure-up to values higher than the ones for T-mixers. Thus, it appears that the value of the tilting angle does influence not only the onset of the engulfment regime but also the mixing behavior within this regime. On the one hand, the increase in $\alpha$ is beneficial to decrease the Reynolds number for the onset of the engulfment regime, but, on the other hand, the mixing performances deteriorate for some values of $R e$ within this regime.

The present work is motivated by the results of Mariotti et al., ${ }^{2}$ and it is aimed at completing the investigation of the mixing behavior of arrow-mixers for larger Reynolds numbers, for which the unsteady periodic flow is usually present in classical T-mixers. ${ }^{10,28-31}$ For T-mixers having inlet channel sections with aspect ratio equal or close to one, the first periodic regime occurring by increasing the Reynolds number after the steady engulfment one is the so-called "unsteady asymmetric regime," 29,30 which is interesting from a practical viewpoint because it is characterized by better mixing than the engulfment one. ${ }^{28,31}$ By further increasing the Reynolds number, the so-called "unsteady symmetric regime" takes place, ${ }^{29}$ which is again periodic in time but is mostly characterized by a symmetric topology in space; this is detrimental to mixing, and indeed, the onset of the unsteady symmetric regime is associated with a dramatic drop of mixing. ${ }^{31}$ The issues herein are whether arrow-mixers are characterized by the same behavior with increasing $R e$, i.e., the onset of the unsteady asymmetric and symmetric regimes, and whether the flow dynamics and the mixing performances are affected by the tilting angle also in the unsteady regimes. In particular, two values of the tilting angle, $\alpha$, viz. $\alpha=10^{\circ}$ ("small" tilting angle) and $\alpha=20^{\circ}$ ("large" tilting angle), which were found in the work of Mariotti et al. ${ }^{27}$ to be characterized by noticeably different flow behavior and mixing in the steady engulfment regime, are considered. A detailed and systematic analysis of the flow behavior and the mixing performance is carried out by considering $R e$ from the ending of the engulfment regime up to the onset of chaotic flow. Numerical simulations are carried out for both considered values of the tilting angle; the numerical results are integrated with experimental flow visualizations for $\alpha=20^{\circ}$. The mixing performances of the two arrow-mixer configurations in the unsteady periodic flow regimes up to the chaotic regime are fully characterized, and thanks to the synergic use of experimental evidence and numerical results, the observed behavior is explained in terms of flow characteristics and physics.

\section{PROBLEM DEFINITION AND INVESTIGATION METHODOLOGIES}

\section{A. Arrow-mixer geometries}

The considered geometries are two of those investigated in the work of Mariotti et ll $^{27}$ and consist of two inlet channels joining in an inclined junction [see Fig. 1(a), which also reports the adopted frame of reference]. We consider herein two different angles between the $x$ axis and the axis of the inlet channels: $\alpha=10^{\circ}$ and $\alpha=20^{\circ}$. For both cases, the inlet channels have a square cross section, with $W=H=1 \mathrm{~mm}$, while the cross section of the mixing channel is a $2: 1$ rectangle, i.e., $W_{o}=2 H=2 \mathrm{~mm}$. Consequently, the mixing channel hydraulic diameter is $d=4 H / 3$. This quantity is used for the definition of the non-dimensional coordinates, $X=x / d, Y=y / d$, and $Z=z / d$. Both inlet channels have a length equal to $L_{i}=40 \mathrm{~mm}$ $\approx 30 d$ such that the flow at the confluence is fully developed, while the mixing-channel length is $L_{o}=60 \mathrm{~mm} \approx 45 d$.

\section{B. Experimental setup}

The experimental model, shown in Figs. 1(b) and 1(c), is made of polymethylmethacrylate (PMMA) and has a tilting angle of $\alpha$ $=20^{\circ}$. The experimental setup is the same as in the work of Mariotti et al., ${ }^{27}$ and it is briefly summarized herein. A syringe pump (KD Scientific) feeds the mixer inlets with deionized water. The two streams entering the device have equal flow rates and, thus, equal bulk velocity, $U$. The food colorant E124 is used to color one inlet stream. The dye is dissolved in water with a concentration of $0.115 \mathrm{wt}$. \%. An upright microscope (Nikon, model Eclipse 80i), having a magnifying lens of $4 \times($ aperture N.A. $=0.13$ ) and a D-LH $12 \mathrm{~V}-100 \mathrm{~W}$ halogen lamp, is used to observe the mixing inside the mixer. We use a high-speed camera (Optomotive Velociraptor HS) to acquire the images with a resolution of $920 \times 2048$ pixels and an acquisition frequency of 387 frames/s. The image exposure time is significantly shorter than the one related to the frame rate, i.e., $\delta T=10^{-6} \mathrm{~s}$. An offline procedure enables the post-processing of the flow visualizations. Lambert-Beer's law allows converting light intensity into normalized depth-averaged dye concentration for each pixel of the image. Additional details on the calibration procedure, the image 


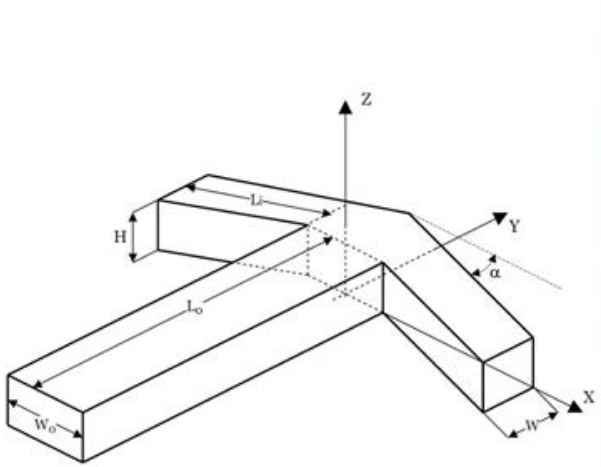

(a)



(b)

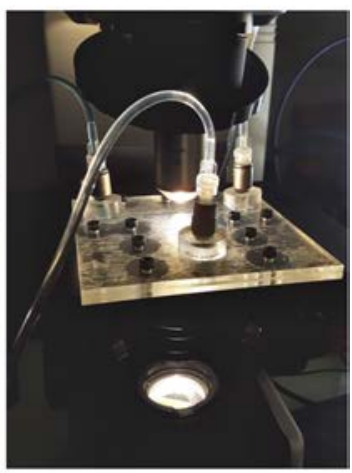

(c)

FIG. 1. Arrow-mixer geometry (a), experimental model (b), and experimental setup (c).

post-processing, and the whole experimental setup are available in the work of Mariotti et al. ${ }^{32}$

\section{Simulation setup}

The unsteady incompressible Navier-Stokes equations in the non-dimensional form are considered to describe the liquid motion. The mixing-channel hydraulic diameter, $d$, is used as a reference length, while the bulk velocity, $U$, is the reference velocity. The Reynolds number is hence defined as $R e=\rho U d / \mu$, where $\rho$ and $\mu$ are the operating liquid density and dynamic viscosity, respectively.

Moreover, the following non-dimensional transport equation describes the dye dynamics:

$$
\frac{\partial \phi}{\partial \theta}+\mathbf{u} \cdot \nabla \phi=\frac{1}{P e} \nabla^{2} \phi
$$

where $\phi$ is the mass fraction of the dye, $\theta$ is the non-dimensional time, $\mathbf{u}$ is the non-dimensional velocity of the fluid, and $P e=U d / D$ is the Peclet number (where $D$ is the molecular diffusivity of the dye).

The numerical methodologies and computational setup are the same as used in the work of Mariotti et al. ${ }^{27}$ and are briefly recalled herein. The computational code Nek5000 ${ }^{33}$ is used for the numerical simulations; it is based on a high-order spectral-element method. The computational domain is divided into hexahedral elements, and Nth-order Lagrange polynomial interpolants, based on tensor-product arrays of Gauss-Lobatto-Legendre (GLL) quadrature points, are used to span the velocity space in each element. A third-order backward differential formula is used for time discretization together with a third-order explicit extrapolation for the convective terms, while the diffusive terms are treated implicitly.

As previously mentioned, two different geometries are considered in the simulations that differ only for the value of the tilting angle, i.e., $\alpha=10^{\circ}$ and $\alpha=20^{\circ}$; for the configuration having $\alpha=20^{\circ}$, experiments are also carried out (see Sec. II B). The simulation domain is the same as in the experiments, except for the lower length of the inlet channels, i.e., $L_{i}=6.875 d$, and of the mixing channel, $L_{o}=25 d$. To have a sensible comparison, a unidirectional fully developed velocity profile is set at the inlets. ${ }^{10}$ As for the mixing channel, the sensitivity analysis presented in the work of Andreussi et al. ${ }^{10}$ for a $\mathrm{T}$ geometry shows that the chosen value of $L_{o}$ together with boundary conditions of free outflow-by assuming that the normal gradient is zero in the $x$ and $z$ directions for the velocity components and that normal stresses are zero-allows spurious effects related to the domain truncation to be avoided. Finally, no-slip is imposed on the mixer walls.

The computational grid has 15680 elements. On the cross sections of the inlet and mixing channels, the elements have a uniform size of $0.075 d$ in both directions. The element size varies between $0.08 d$ and $0.5 d$ along the axis of the channels. The polynomial order for the velocity is fixed to $N=6$, while for the pressure, the order is $N-2=4$, thus using the common $P_{N} / P_{N-2}$ approach. The degrees of freedom for each velocity component are approximately $10^{7}$. The time step is $\Delta t=7.0 \cdot 10^{-4} \frac{d}{U}$, resulting in a Courant-Friedrichs-Lewy number $C F L=\frac{\Delta t u}{\Delta x} \leq 0.3$, with $\Delta x$ being the size of the computational cell. Stability issues force to use a rather low Schmidt number, i.e., $S c=\mu /(\rho D)=O(100)$ in the scalar equation. ${ }^{30}$ Thus, the Schmidt number is one or two orders of magnitude lower than the typical values for liquids. Nevertheless, this enhanced diffusion has a negligible effect on the confluence region analyzed in the present work because of the strong transversal convection, as demonstrated in the work of Galletti et al. ${ }^{34}$ for a T-shaped micro-mixer.

Uniform non-developed flow conditions are chosen to initialize all the simulations to prevent hysteresis phenomena. ${ }^{10,11}$ Simulations are run for a minimum number of 20 periodic cycles, after having discarded the initial transient. The numerical data are analyzed to compute the depth-averaged dye concentration and directly compare this quantity with the one measured in the experiments. ${ }^{27}$ The inlet dye concentration is used to obtain non-dimensional values of the local dye concentration, which thus ranges from 0 to 1 . Moreover, the degree of mixing, $\delta_{m}$, is used to have a numerical quantification of the mixing between the two streams. For a given cross section in the mixing channel, $\delta_{m}$ is computed as follows:

$$
\delta_{m}=1-\frac{\sigma_{b}}{\sigma_{\max }}
$$


where $\sigma_{b}$ is the standard deviation of the volumetric flow, having the following expression:

$$
\sigma_{b}^{2}=\frac{\int\left(\phi-\bar{\phi}_{b}\right)^{2} u_{y} d x d z}{\int u_{y} d x d z}
$$

where $u_{y}$ is the velocity component in the $y$ direction and $\bar{\phi}_{b}$ is the bulk mass fraction evaluated as

$$
\bar{\phi}_{b}=\frac{\int \phi u_{y} d x d z}{\int u_{y} d x d z} .
$$

Finally, $\sigma_{\max }$ is the maximum value of $\sigma_{b}$, which is obtained for two completely segregated streams,

$$
\sigma_{\max }=\sqrt{\bar{\phi}_{b}\left(1-\bar{\phi}_{b}\right)} .
$$

Consequently, $\delta_{m}$ varies in the range $0 \leq \delta_{m} \leq 1$. A completely segregated flow leads to $\delta_{m}=0$, whereas a fully mixed flow leads to $\delta_{m}=1$.

In the numerical simulations, the vortical structures are visualized by means of the $\lambda_{2}$ criterion. ${ }^{35}$ According to this criterion, a vortex is defined as a connected fluid region where the second largest eigenvalue, $\lambda_{2}$, of the symmetric tensor $\mathbf{L}=\mathbf{S} \cdot \mathbf{S}+\mathbf{A} \cdot \mathbf{A}$ is negative, where $\mathbf{S}$ and $\mathbf{A}$ indicate the symmetric and anti-symmetric part of the velocity gradient, which are the strain rate and vorticity tensors, respectively. In addition to $\lambda_{2}$, many other different criteria can be used for the identification of a vortical structure, viz. $Q$-criterion, $\Delta$-criterion, and $|\omega|$-criterion. ${ }^{35}$ We preliminary compared the $3 \mathrm{D}$


(a)
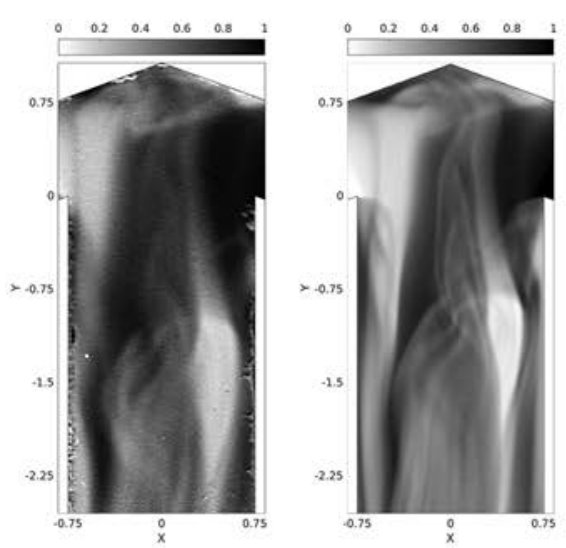

(c)
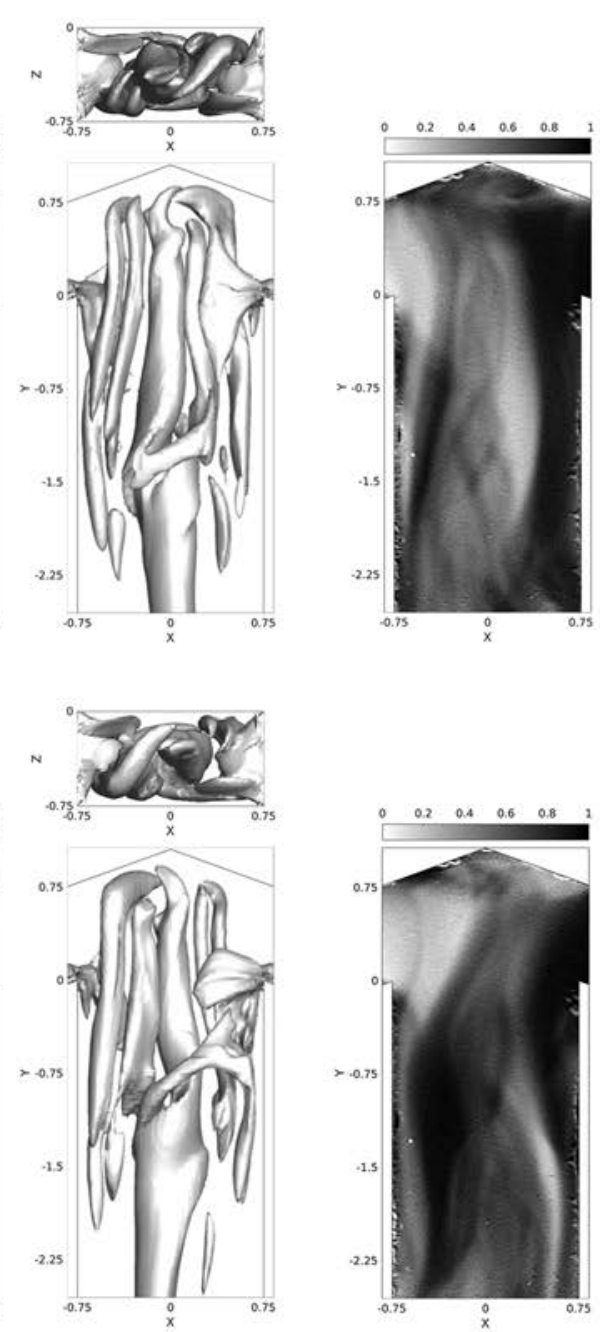
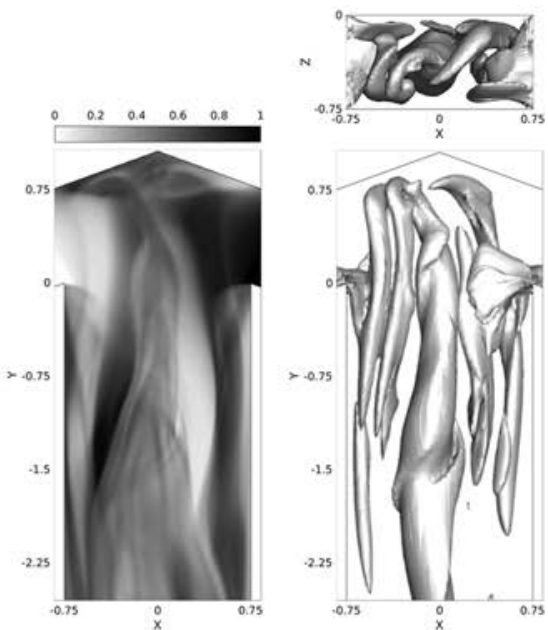

(b)
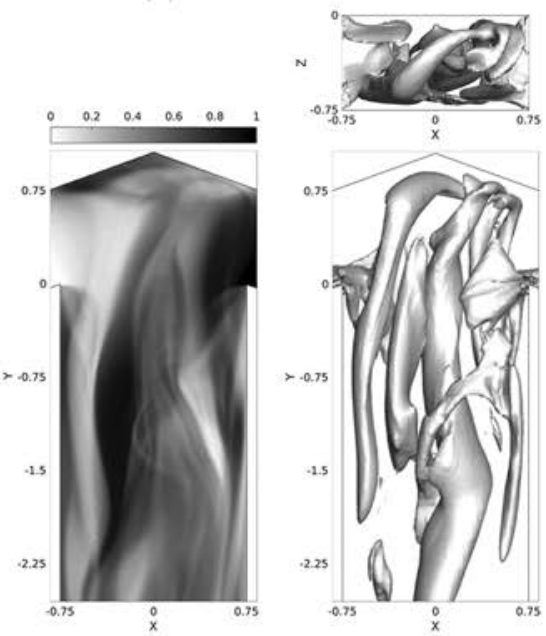

(d)

FIG. 2. Experimental image (left), CFD depth-averaged dye concentration field (middle), and isosurface of the $\lambda_{2}$ vortex indicator (right) at $R e=240$ at different times: (a) $t / \tau=0$, (b) $t / \tau=0.25$, (c) $t / \tau=0.5$, and (d) $t / \tau=0.75$. Multimedia view: https://doi.org/10.1063/5.0033765.1 
vortical structures given by the $\lambda_{2}$ indicator with those obtained by the $Q$-criterion, i.e., the region of positive $Q=\frac{1}{2}\left(\|\mathbf{A} \cdot \mathbf{A}\|^{2}-\|\mathbf{S} \cdot \mathbf{S}\|^{2}\right)$ $=-\frac{1}{2}\left(\lambda_{1}+\lambda_{2}+\lambda_{3}\right)$, where $\lambda_{1} \geq \lambda_{2} \geq \lambda_{3}$ are the three eigenvalues of $\mathbf{L}$. The identified vortical structures were practically identical, and thus, we decided to use the $\lambda_{2}$ vortex indicator in the present paper.

Finally, the frequencies $f$ in the periodic flow regimes for both experiments and numerical simulations are made non-dimensional with the hydraulic diameter of the mixing channel and the inlet bulk velocity and, thus, presented in terms of Strouhal number, $S t=\frac{f d}{U}$, in Secs. III-V.

\section{FLOW REGIMES FOR THE ARROW-MIXER WITH $\alpha=20^{\circ}$}

In this section, we present the results of the experimental flow visualizations and the numerical simulations obtained for different Reynolds numbers in the arrow-mixer having $\alpha=20^{\circ}$.



(a)

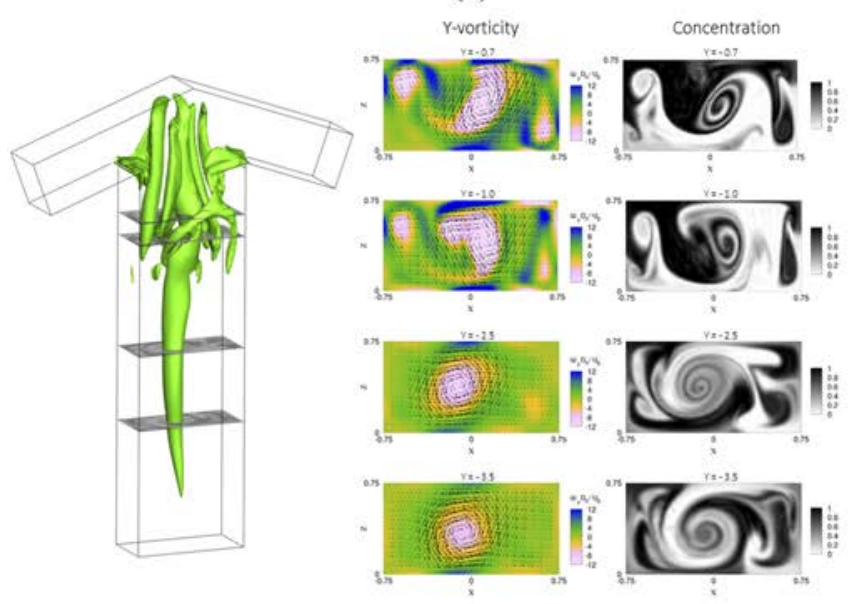

(c)

\section{A. Periodic regime with a single vortex}

As described in the work of Mariotti et al., ${ }^{27}$ in the range $R e$ $=[170-190]$, the flow is steady and characterized by a single strong vortical structure at the center of the mixing channel. As previously mentioned, this flow topology is not present for T-mixers and lower tilting angles. At $R e=200$, this vortex starts to oscillate periodically in time, and the oscillation amplitude increases with $R e$, reaching the maximum value at about $R e=240$. Figure 2 (Multimedia view) shows the experimental flow visualizations in the left panels and the corresponding numerical depth-averaged dye concentration fields for four different time instants spanning a whole characteristic cycle in time at $R e=240$ in the middle panels. The video provides a further view of the flow dynamics. Experimental flow visualizations and numerical simulations agree very well qualitatively, and the signatures of the single vortical structure are visible. The vortical structure is visualized in the right panels of Fig. 2 (Multimedia view) by means of the $\lambda_{2}$ criterion. ${ }^{35}$ The same $2 \mathrm{D}$ view of the $3 \mathrm{D}$ isosurface of $\lambda_{2}$ as
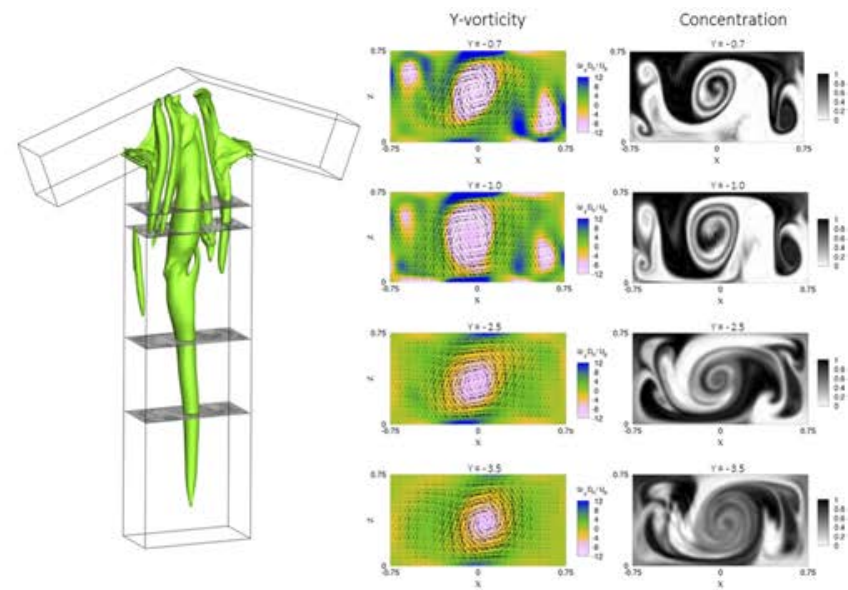

(b)

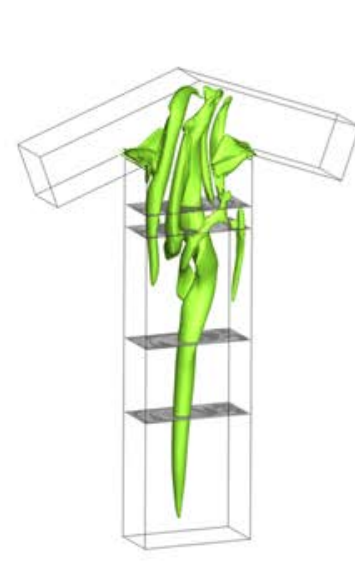

(d)

FIG. 3. Isosurface of the $\lambda_{2}$ vortex indicator from a different view (left), $y$-vorticity and in-plane velocity (middle), and dye concentration fields (right) at $R e=240$. Considered cross sections (from top to bottom): $Y=-0.7, Y=-1, Y=-2.5$, and $Y=-3.5$. Simulation times: (a) $t / \tau=0$, (b) $t / \tau=0.25$, (c) $t / \tau=0.5$, and (d) $t / \tau=0.75$. Multimedia view: https://doi.org/10.1063/5.0033765.2 
in the dye visualizations is shown in the bottom right panel, together with a top view in the top right panel. The $3 \mathrm{D}$ view of the isosurface of $\lambda_{2}$ is shown in the left panel of Fig. 3 (Multimedia view) with the contours of $y$-vorticity and in-plane velocity (middle panel) and the dye concentration fields (right panel) in different cross sections of the mixing channel and at the same time instants as in Fig. 2 (Multimedia view).

The large vortical structure in the center of the mixing channel is visible [see the right panels of Fig. 2 (Multimedia view) and the left panels of Fig. 3 (Multimedia view)]. It can be seen from the dye distribution in the different cross sections of the mixing channel [Fig. 3 (Multimedia view)] how the central vortical structure promotes mixing between the two streams through a spiral pattern. Moreover, both Figs. 2 and 3 (Multimedia view) show that this vortical structure is not steady in time; indeed, from the middle and right panels of Fig. 3 (Multimedia view), it can also be seen that it moves along the $x$ and $z$ directions. This kind of dynamics is not observed in $\mathrm{T}$-mixers, in which the first periodic regime is dominated by the

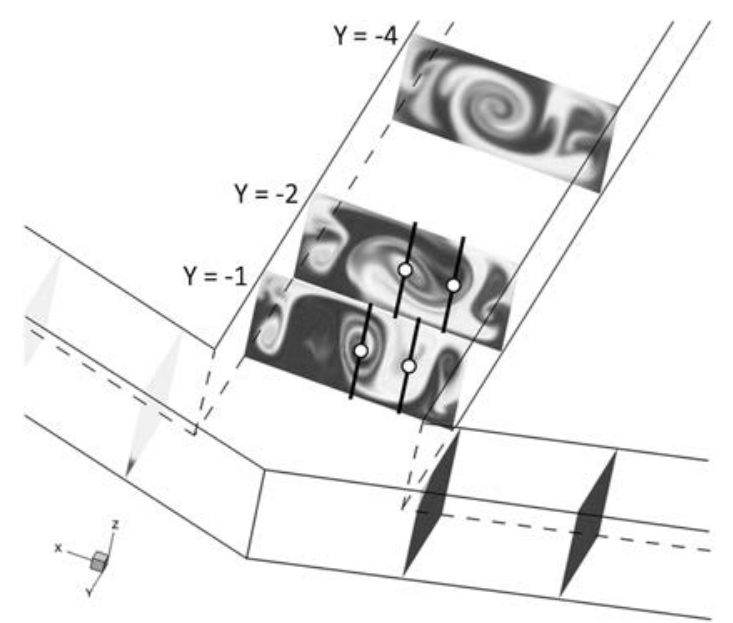

(a)
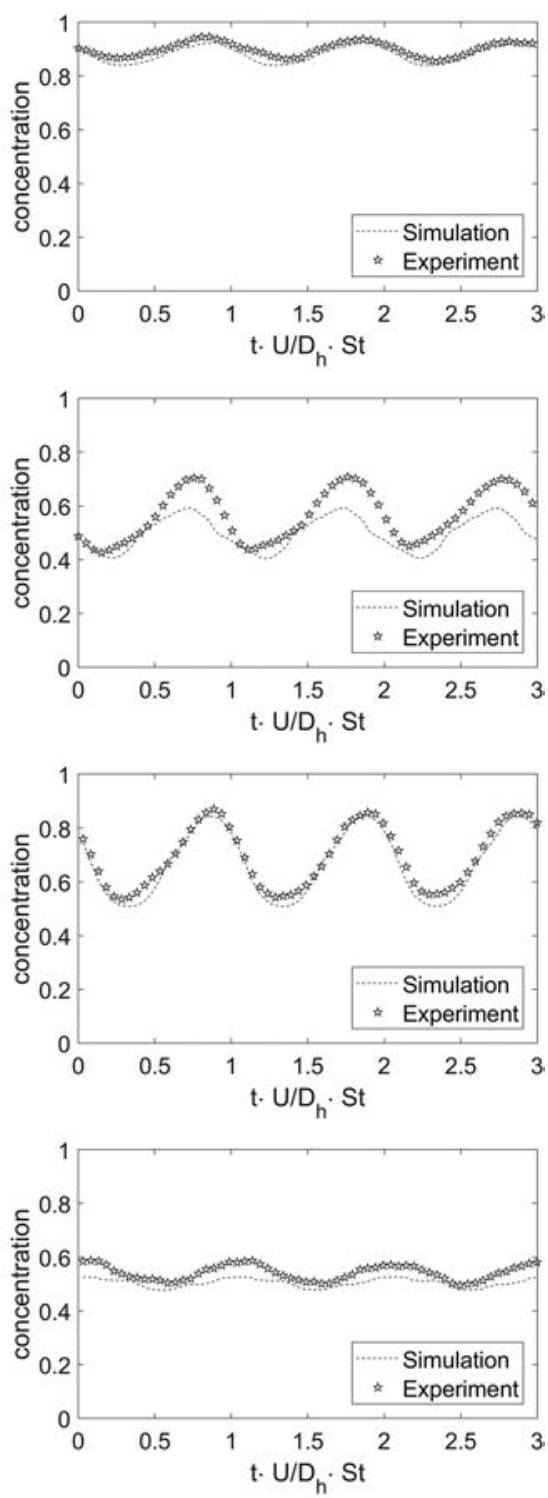

(b)

FIG. 4. Dye concentration field at different cross sections in the arrow-mixer and position of the considered probes indicated with white markers and black lines (a). Experimental and numerical time behavior of the depth-averaged dye concentration at (from top to bottom) $(X, Y)=(-0.375,-1),(0,-1),(-0.375,-2),(0,-2)(b)$. 
tilting and the motion of the top parts of the two 3D vortical structures-also present in the steady engulfment regime-which leads to the periodic shedding of a vorticity blob in the mixing channel (see, e.g., Refs. 10, 30, and 32 and the description that will be given in Sec. IV A of the present paper).

To further investigate the periodicity of the flow dynamics and check the agreement between experiments and simulations, Fig. 4 shows four temporal signals of the dye concentration, averaged in the channel depth, obtained in experiments and simulations at the locations shown in the same figure. For all the considered locations, the behavior is periodic and the period, $\tau$, gives a Strouhal number equal to $S t=0.244$ in the simulation, which is slightly larger than that obtained in the experiment, $S t=0.218$. Despite this small difference in the frequency value, the shape of the dye signal in experiments and simulations is in good agreement, if they are plotted as a function of $t / \tau$ as in Fig. 4(b). Some minor discrepancies may be partly imputed to the fact that, for numerical stability issues, a rather low Schmidt number, i.e., $S c=\mu /(\rho D)=O(100)$, is used in the scalar equation (see Sec. II C) in the simulations. Thus, the Schmidt number is one or two orders of magnitude lower than the typical values for liquids. As demonstrated in the work of Galletti et al. ${ }^{34}$ for a T-shaped micro-mixer, this enhanced diffusion has a negligible effect on the global evaluation of the flow field in the confluence region, but it is reasonable to infer that it may give a sort of slight smoothing in the punctual depth-averaged dye concentration signals. This may be the cause of the slightly reduced variance of the numerical signals compared to the experimental ones. The matching between the experimental and numerical signal profiles is consistent with the one previously found for the dye-concentration signals in T-shaped micro-mixers in the periodic asymmetric and the periodic symmetric flow regimes (see Figs. 5 and 7 of Ref. 31).

\section{B. Steady symmetric regime}

Interestingly, at Reynolds numbers $R e=260$ and $R e=280$, the flow becomes steady again both in experiments and simulations; moreover, its topology appears very similar to that in the vortex regime. The flow features at $R e=280$ are described herein in more details; those at $R e=260$ are practically the same. Qualitative agreement between the depth-averaged dye concentration field from experiments and simulations is once again really good [compare Fig. 5(a) with Fig. 5(b)]. The two flow streams remain segregated in the mixing channel, in which four vortical structures are present [see the isosurface of the vortex indicator $\lambda_{2}$ in Figs. 5(c) and 6(a)]. These vortices are the legs of the two structures forming in the top part of the mixer at the confluence of the two inlet streams, which are in turn related to the flow recirculations occurring near the top walls. This flow pattern is very similar to that described in the work of Mariotti et al. ${ }^{27}$ for the vortex regime at $R e=120$ (compare Figs. 5 and 6 with Figs. 2 and 4 of Ref. 27). The described flow topology has two reflectional geometrical symmetries in the mixing channel; this prevents convection from contributing to mixing,
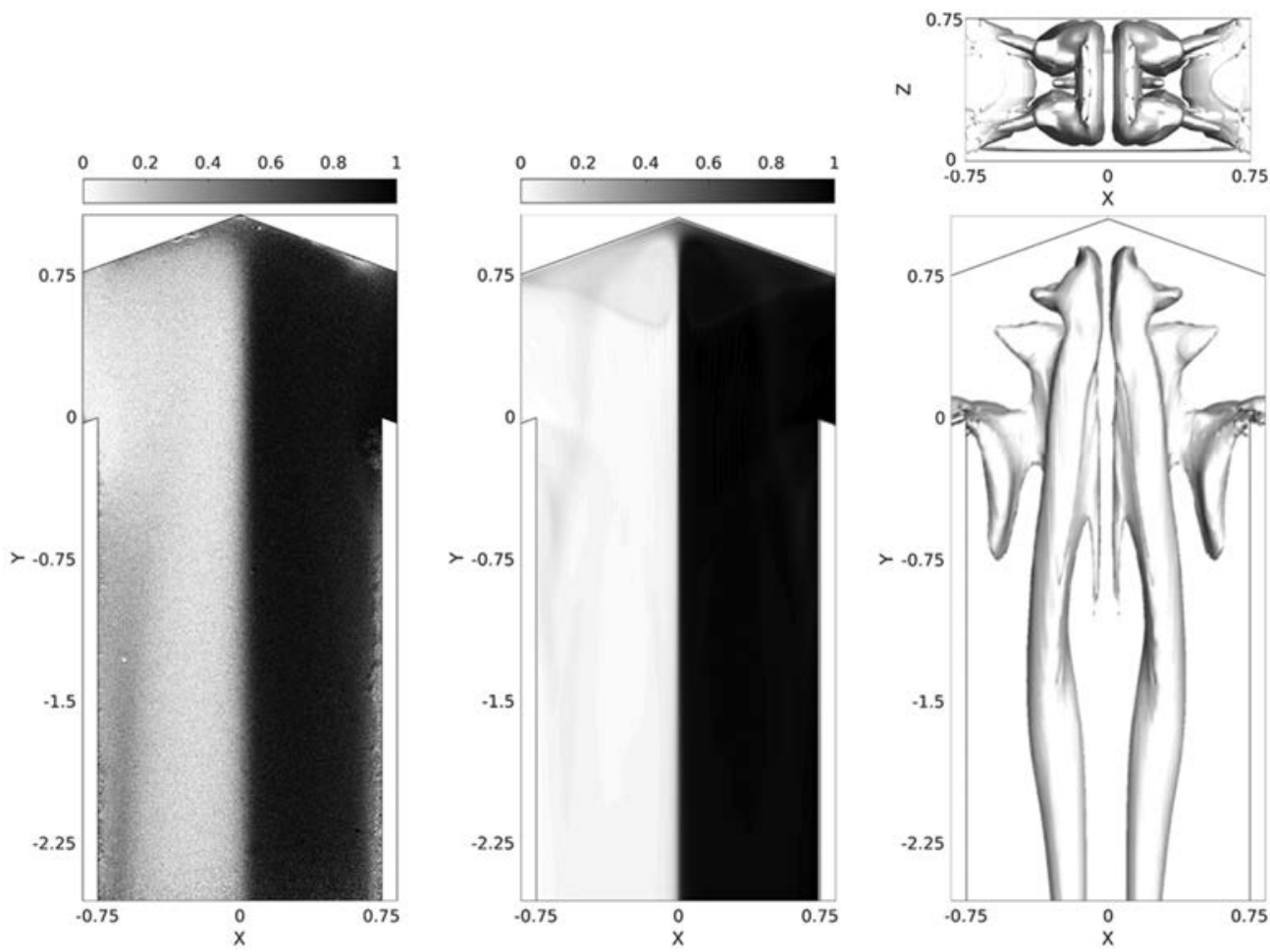

FIG. 5. Experimental image (left), CFD depth-averaged dye concentration field (middle), and isosurface of the $\lambda_{2}$ vortex indicator (right) at $R e=280$. 




FIG. 6. Isosurface of the $\lambda_{2}$ vortex indicator from a different view (left), $y$-vorticity and in-plane velocity (middle), and dye concentration fields (right) at $R e=280$. Considered cross sections (from top to bottom): $Y=-0.7, Y=-1, Y=-2.5$, and $Y=-3.5$.
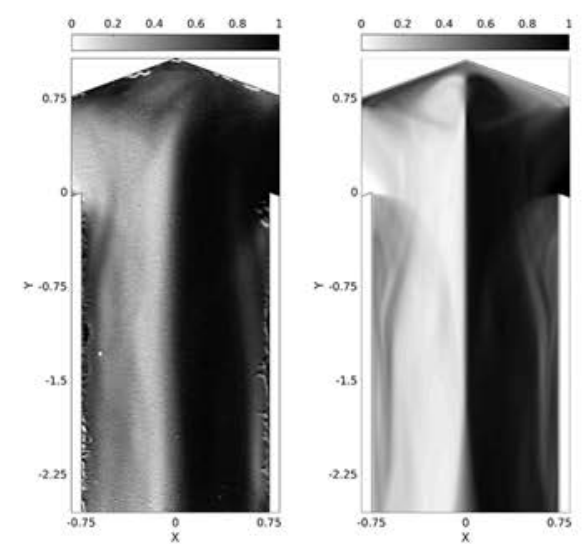

(a)

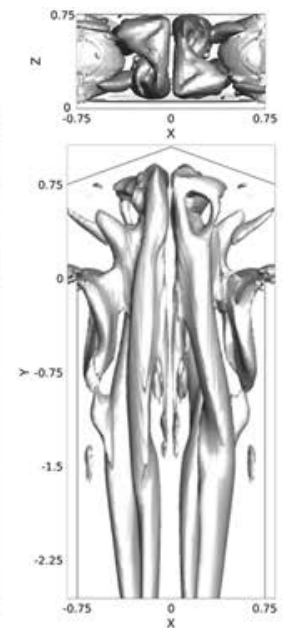

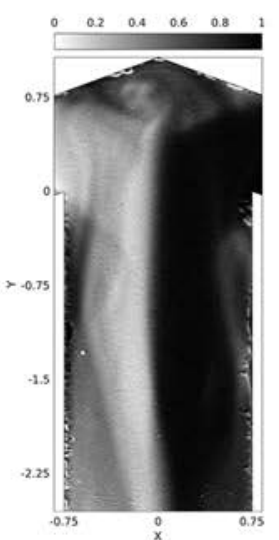

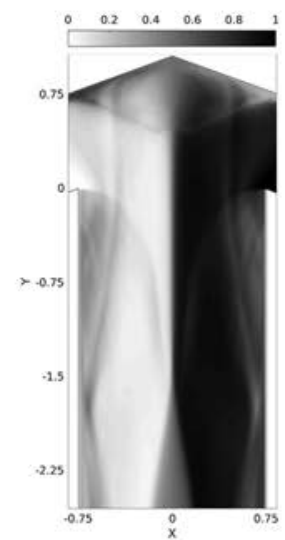

(b)

FIG. 7. Experimental image (left), CFD depth-averaged dye concentration field (middle), and isosurface of the $\lambda_{2}$ vortex indicator (right) at $R e=320$ at different times: (a) $t / \tau=0$ and (b) $t / \tau=0.5$. Multimedia view: https://doi.org/10.1063/5.0033765.3 


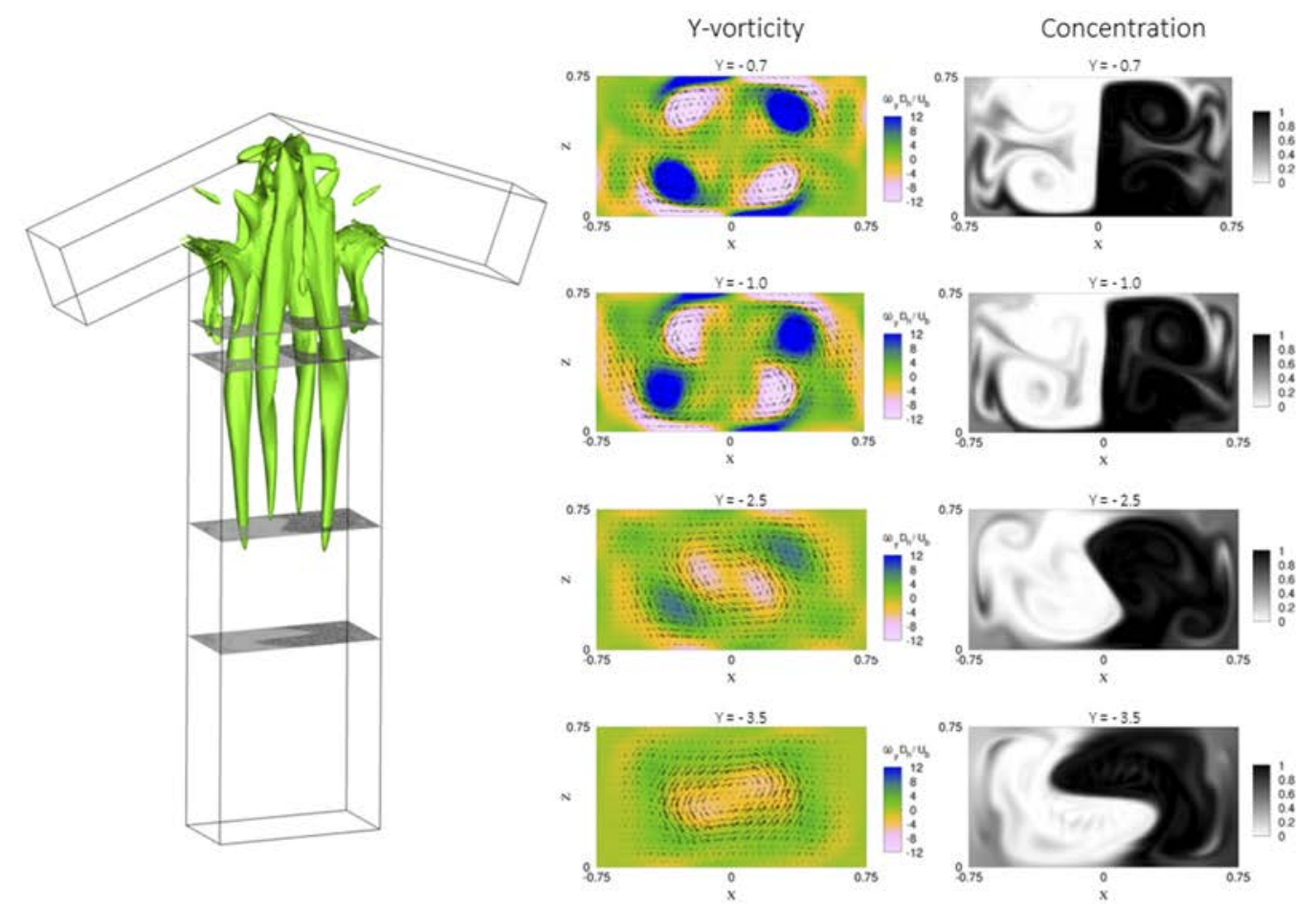

(a)

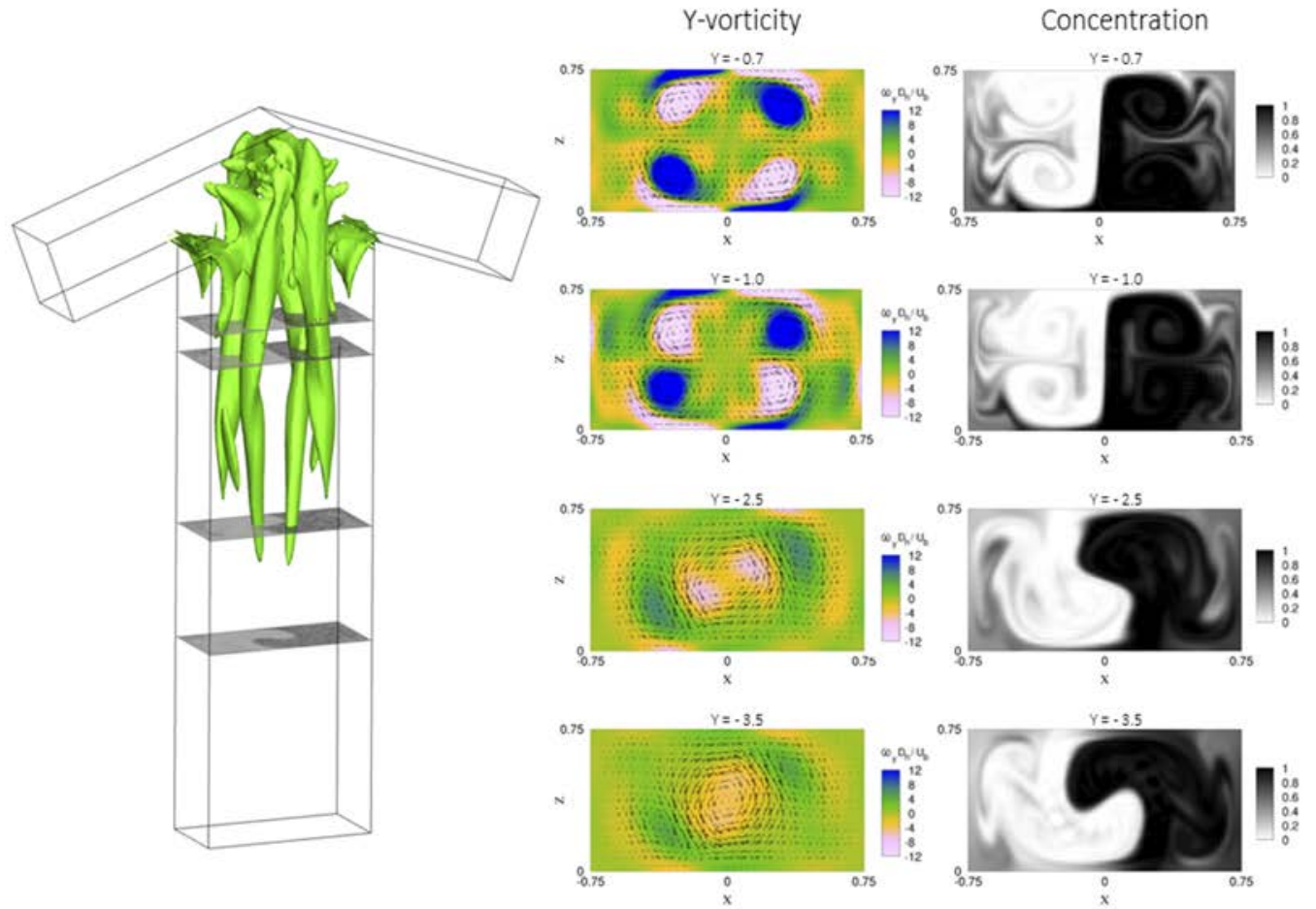

(b)

FIG. 8. Isosurface of the $\lambda_{2}$ vortex indicator from a different view (left), $y$-vorticity and in-plane velocity (middle), and dye concentration fields (right) at $R e=320$. Considered cross sections (from top to bottom): $Y=-0.7, Y=-1, Y=-2.5$, and $Y=-3.5$. Simulation times: (a) $t / \tau=0$ and (b) $t / \tau=0.5$. Multimedia view: https://doi.org/10.1063/5.0033765.4 
which occurs only through diffusion. Consequently, the two streams remain practically unmixed also in the mixing channel. It can be easily understood that the onset of this regime corresponds to a drop in the degree of mixing. This will be quantified and discussed in Sec. V.

\section{Periodic almost symmetric regime}

By further increasing the Reynolds number, for $R e=300$ and $R e=320$, the flow becomes once again unsteady and periodic in time. Let us consider, for instance, the results obtained at $R e=320$, shown in Figs. 7 and 8. Each period presents a slow tilting of the top parts of the 3D vortical structures from a configuration, in which they are almost perpendicular to the incoming streams [see Fig. 7(a) at time $t / \tau=0$ as we arbitrarily choose this pattern as the beginning of the cycle] to a maximum tilting angle of $\simeq 45^{\circ}$ for $t / \tau=0.5$ [Fig. 7(b)]. Despite this quite large rotation, the flow in the mixing channel is not too much affected [compare Figs. 8(a) and 8(b)]. Indeed, two couples of counter-rotating vortices, which are the two legs of the two $3 \mathrm{D}$ vortical structures, are always present in the first portion of the mixing channel. In this first part of the mixing channel, the flow maintains a central symmetry around the channel axis, and thus, the two streams remain almost segregated [see the top two middle and right panels of Figs. $8(\mathrm{a})$ and $8(\mathrm{~b})$ ]. The two legs containing positive $\omega_{y}$ vorticity gradually disappear when moving downwards, while the ones with negative vorticity merge. In this region, mixing between the two streams starts to occur, thanks to flow convection [see the bottom two middle and right panels of Figs. 8(a) and 8(b)]. The periodic tilting of the top parts of the 3D vortical structures only changes the location at which these phenomena occur; when the top parts are tilted, the vanishing and merging of the legs take place more upstream [see Fig. 8 (Multimedia view)].

To evaluate the characteristic frequency of this periodic dynamics, the more suitable locations for evaluating the depth-averaged dye concentration signals are those on the top of the mixer, where the rotation of the vortical structures produces a sudden variation of the dye concentration. For instance, the numerical and experimental temporal signals of the depth-averaged dye concentration, at the location indicated by the thick line in Fig. 9(a), are compared in Fig. 9(b). Both experimental and numerical signals are periodic, following the rotation of the top part of the vortical structure. In addition, in this case, the characteristic Strouhal number obtained in the simulation, $S t=0.367$, is somewhat larger than the experimental one, $S t=0.325 \pm 0.02$. However, if we plot the signals as a function of $t / \tau$, the behavior obtained in experiments is in a good agreement with that given by numerical simulations.

\section{UNSTEADY REGIMES FOR THE ARROW-MIXER WITH $\alpha=10^{\circ}$}

We describe herein the unsteady flow regimes occurring in an arrow-shaped micro-mixer having $\alpha=10^{\circ}$. We carried out only numerical simulations for this configuration.

\section{A. Periodic asymmetric regime}

For $\alpha=10^{\circ}$, Mariotti et al. ${ }^{27}$ found that the steady engulfment regime, which occurs for $R e \geq 140$, always presents two co-rotating vortices in the mixing channel, leading to a monotonic increase in the degree of mixing, as in T-mixers.

If the Reynolds number further increases, the flow is steady up to $R e=200$. The first unsteady flow regime, observed in the range $R e=[200-300]$, is similar to the periodic asymmetric one observed for T-mixers. ${ }^{31,32}$ Figure 10 (Multimedia view) shows the 3D views of the $\lambda_{2}$ isosurface (left panel), the contours of the $y$-vorticity and in-plane velocity (middle panel), and the dye concentration fields (right panel) evaluated in different cross sections of the mixing channel for $R e=260$. Four equally spaced time instants, representative of the flow dynamics in this regime, are reported. Figure 10(a) has been arbitrarily chosen as the beginning of the flow dynamics cycle $(t / \tau=0)$. The first flow configuration of the cycle is very similar to the engulfment regime (compare it with Fig. 19 of Ref. 27), except for a rotation of the legs in the final part of the mixing channel that is related to the shedding of the vorticity blob in the previous cycle. As time progresses, the top parts of the vortical structures tend to approach each other, and eventually, they merge and collapse in a blob of vorticity that is convected in the mixing channel [see Fig. 10 (b) at $t / \tau=0.25$ ]. In the meanwhile that the vorticity blob flows in the mixing channel, two new vortical structures form

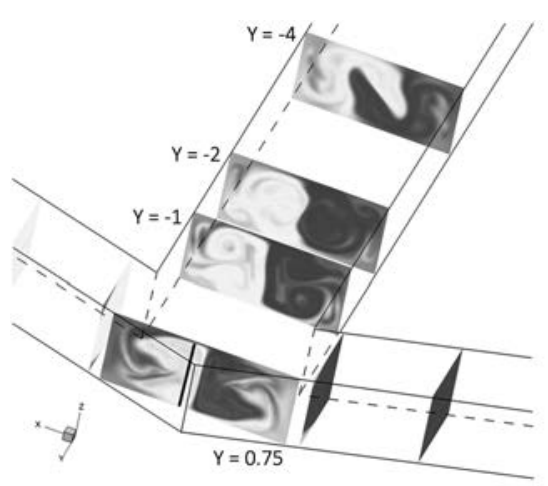

(a)

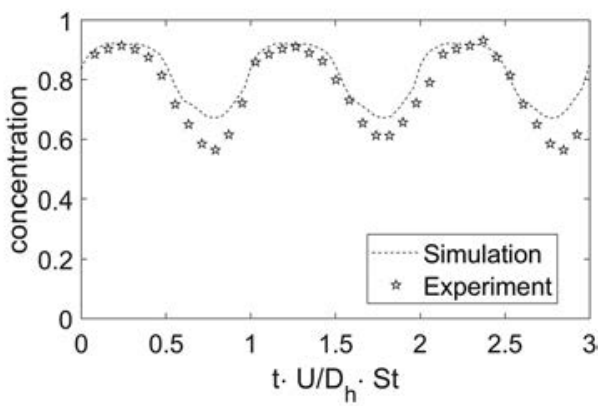

(b)
FIG. 9. Dye concentration at different cross sections in the arrow-mixer and position of the considered line for depthaveraging of the dye concentration (a) Experimental and numerical time behavior of the depth-averaged dye concentration at $(X, Y)=(0.1,0.75)(b)$ 


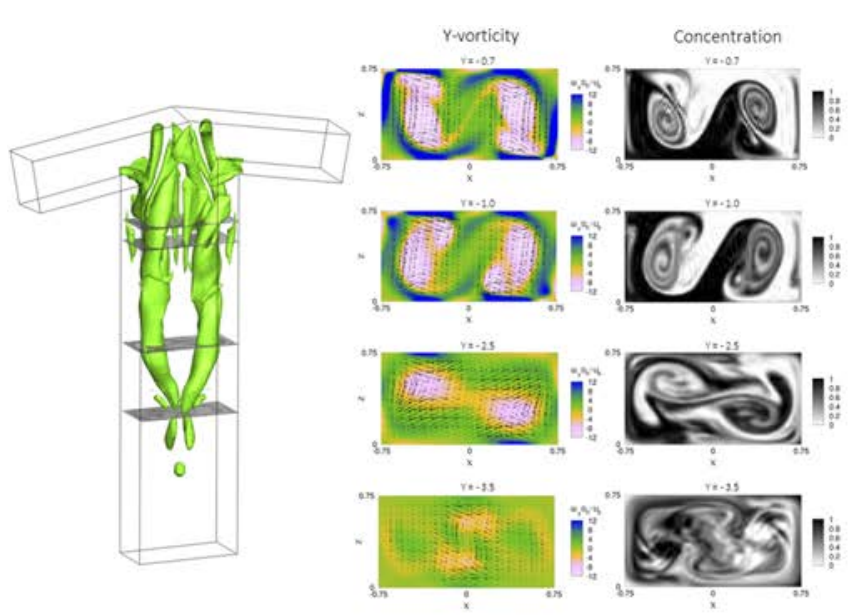

(a)

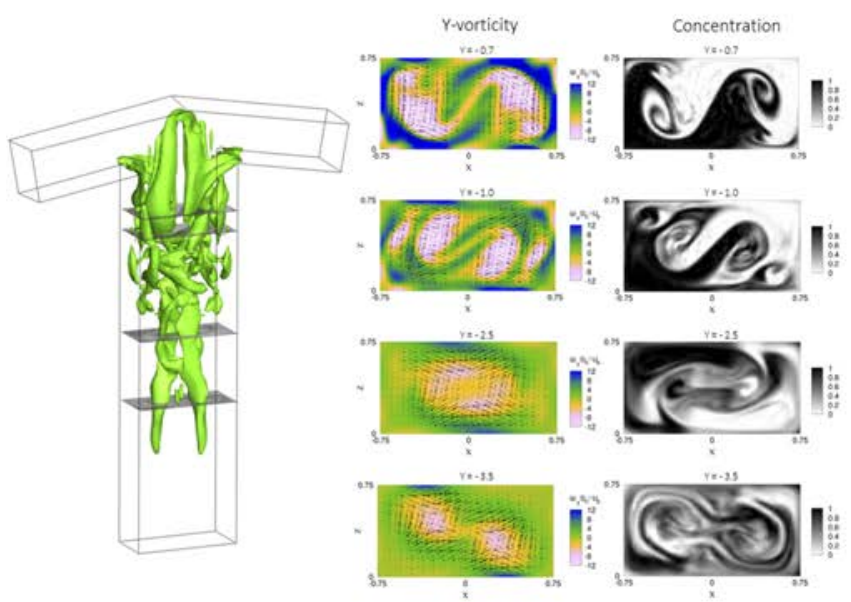

(c)



(b)
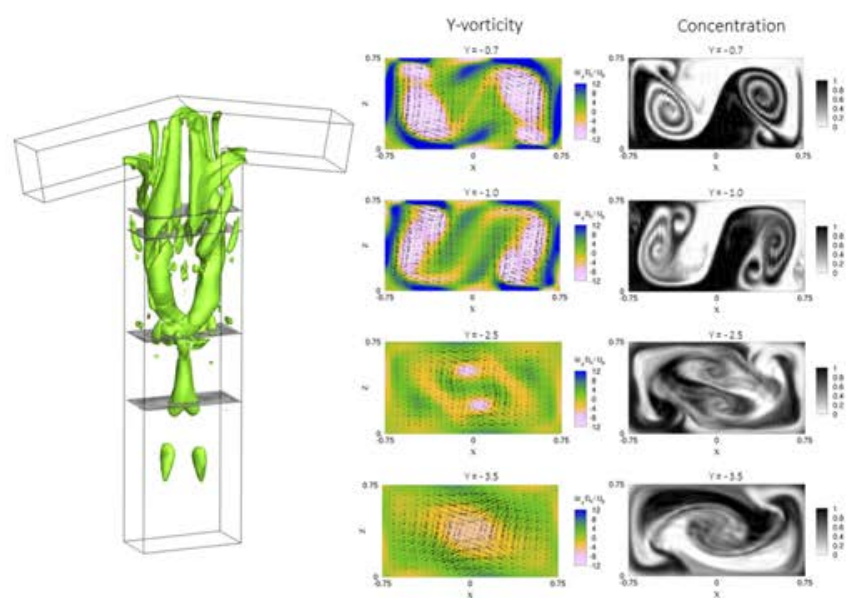

(d)

FIG. 10. Isosurface of the $\lambda_{2}$ vortex indicator from a different view (left), $y$-vorticity and in-plane velocity (middle), and dye concentration fields (right) at $R e=260$. Considered cross sections (from top to bottom): $Y=-0.7, Y=-1, Y=-2.5$, and $Y=-3.5$. Simulation times: (a) $t / \tau=0$, (b) $t / \tau=0.25$, (c) $t / \tau=0.5$, and (d) $t / \tau=0.75$. Multimedia view: https://doi.org/10.1063/5.0033765.5

from both the inlet channels, move toward, and replace the previous ones [see Fig. 10(b) at $t / \tau=0.50$ ]. The passage of the vorticity blob is also visible in the dye distributions in the right panels of Figs. 10(c) and 10(d).

\section{B. Periodic regime with a single vortex}

For further increase in the Reynolds number, the asymmetric periodic regime with vorticity blob shedding, described in Sec. IV A, changes into a regime in which a single strong vortical structure oscillates in the mixing channel. This flow dynamics occurs in the range $R e=[320-400]$, and it is practically the same as the one found for $\alpha=20^{\circ}$ at lower Reynolds numbers (see Sec. III A). We report in Fig. 11 (Multimedia view) the result for a characteristic cycle at $R e=350$. The flow dynamics is similar to the one shown in Fig. 3
(Multimedia view) for the arrow-mixer with $\alpha=20^{\circ}$ at $R e=240$, except for an increase in the intensity of both the single vortex and of the secondary recirculations, which is probably connected to the increased Reynolds number. Thus, analogous considerations as done in Sec. III A are valid also in this case.

\section{MIXING PERFORMANCES}

The mixing between the two streams can be quantified through $\delta_{m}$, defined in Eq. (2). Figure 12 shows $\delta_{m}$ vs $R e$, estimated in the mixing channel at the $Y=-8$ section in the unsteady numerical simulations, for the arrow-mixers with $\alpha=10^{\circ}$ and $\alpha=20^{\circ}$. For completeness, the mixing degrees in the steady flow regimes, reported 

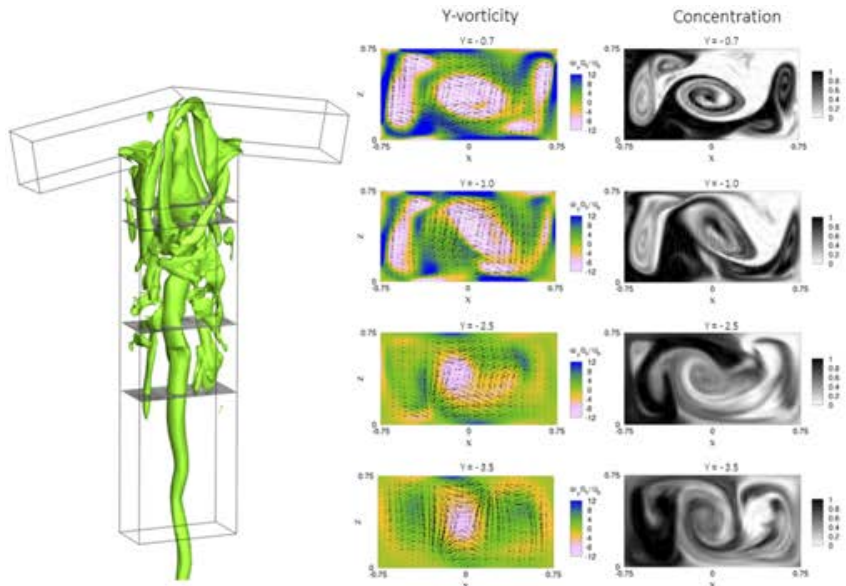

(a)
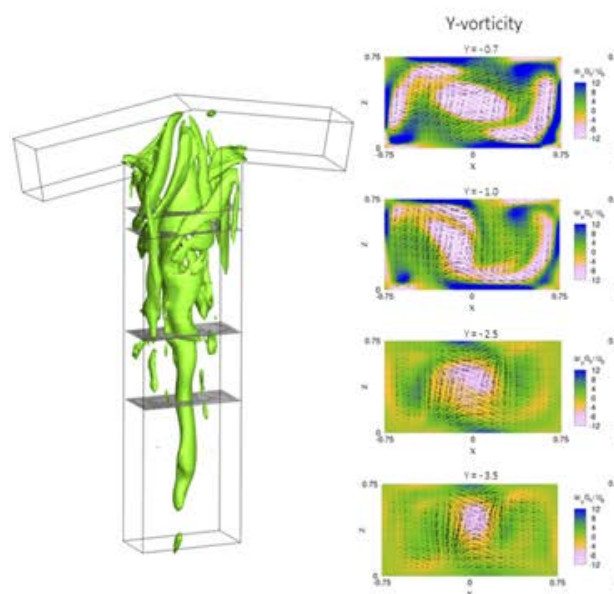

(c)
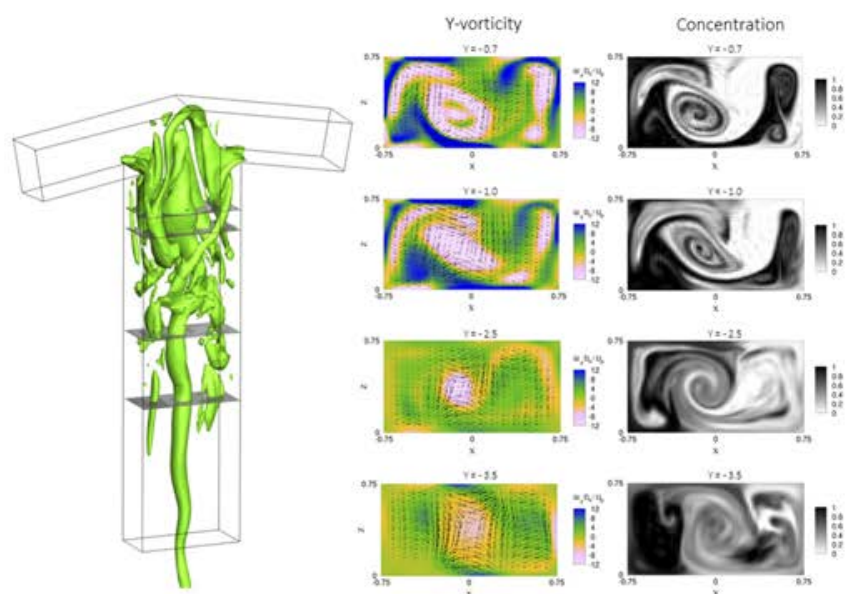

(b)



(d)

FIG. 11. Isosurface of the $\lambda_{2}$ vortex indicator from a different view (left), $y$-vorticity and in-plane velocity (middle), and dye concentration fields (right) at $R e=350$. Considered cross sections (from top to bottom): $Y=-0.7, Y=-1, Y=-2.5$, and $Y=-3.5$. Simulation times: (a) $t / \tau=0$, (b) $t / \tau=0.25$, (c) $t / \tau=0.5$, and (d) $t / \tau=0.75$. Multimedia view: https://doi.org/10.1063/5.0033765.6

in the work of Mariotti et al., ${ }^{27}$ are also shown. Moreover, the curve numerically obtained by using the same methodology for a T-shaped mixer with the same channel aspect ratios $\left(\alpha=0^{\circ}\right)$ and operating at the same conditions ${ }^{31}$ is also reported for comparison. Figure 13 summarizes the steady and unsteady flow regimes occurring in the considered mixers.

Figure 12 allows a quantification of the previous qualitative observations of the mixing behavior in the different regimes. A first sharp increase in the mixing degree is related to the onset of the engulfment regime, which, as shown in the work of Mariotti et al., takes place at lower Reynolds numbers for increased tilting angle $\alpha$ of inlet channels. As already described in the work of Mariotti et al., for $\alpha=20^{\circ}$, a significant drop of mixing is first observed within the steady engulfment regime, which is not present for T-mixers and $\alpha=10^{\circ}$. This drop is related to the previously described change in the flow topology, i.e., to the presence of a single vortical structure at the center of the mixing channel instead of two co-rotating ones, characterizing the engulfment regime in T-mixers. Further increasing $R e$, the flow topology remains the same but mixing augments because of the increase in strength and intensity of the vortical structure in the mixing channel.

We now analyze more in detail the $\delta_{m}$ behavior in the unsteady regimes, which are the focus of the present work, to highlight how mixing is influenced by $\alpha$. Figure 12 shows with symbols the average value of $\delta_{m}$ in a temporal period, while bars indicate the range of $\delta_{m}$ values spanned in the cycle. First, the mixing behavior for $\alpha=0^{\circ}$ (i.e., for a T-mixer), as analyzed in the work of Mariotti et al., ${ }^{31}$ is summarized here. $\delta_{m}$ increases significantly with Reynolds numbers in the so-called periodic asymmetric regime, which is very similar to that occurring for the $\alpha=10^{\circ}$ configuration and described in Sec. IV A. 




FIG. 12. Degrees of mixing numerically evaluated at the cross section $Y=-8$ as a function of the Reynolds number $R e$ and of the tilting angle $\alpha$. The red lines represent data fits following Eq. (6). The data for $\alpha=0$ are from the work of Mariotti et al. ${ }^{31}$

At $R e \simeq 325$, a dramatic drop of $\delta_{m}$ occurs at the transition to the so-called periodic symmetric regime. Indeed, in this regime, the two streams in the mixing channel are again almost segregated (see Ref. 32 for a description).

For $\alpha=20^{\circ}$, the first unsteady regime originates from a steady flow topology characterized by the presence of a single vortical structure in the mixing channel, which is not observed in T-mixers and consists in periodic oscillations of this structure (see Sec. III A). These oscillations are beneficial for mixing; indeed, the maximum

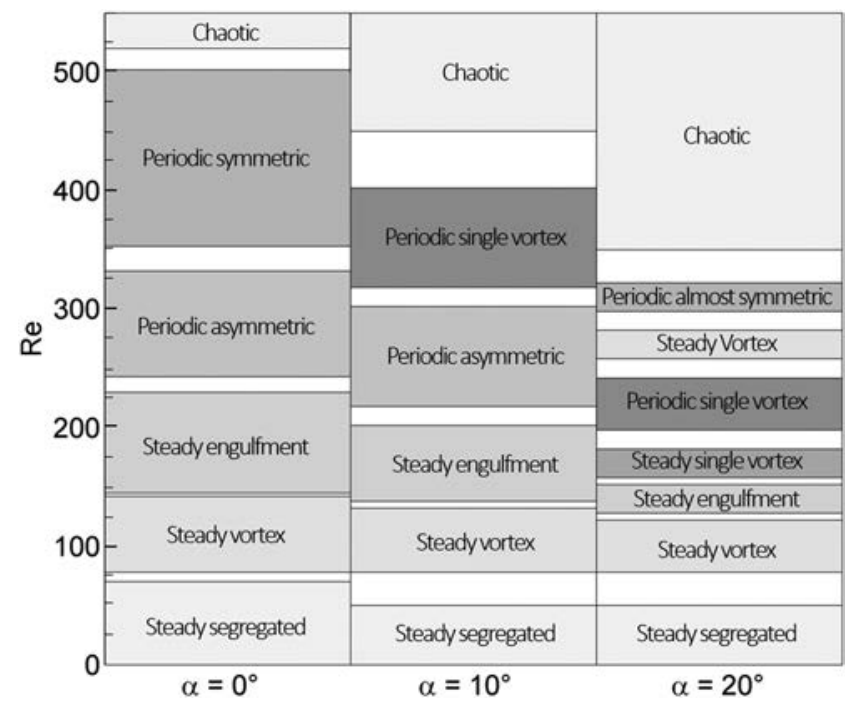

FIG. 13. Summary of the flow regimes as a function of the Reynolds number and of the tilting angle $\alpha$. values of $\delta_{m}$ occur in this periodic regime up to $R e \simeq 240$. Subsequently, another dramatic drop of $\delta_{m}$ to values significantly lower than those of the steady regimes takes place. This drop is due to the unexpected onset of a steady flow regime, which is very similar to the vortical one, as described in Sec. III B. Finally, the flow becomes again periodic in time, showing a flow regime in which the two streams remain almost segregated in a large portion of the mixing channel (see Sec. III C); hence, $\delta_{m}$ increases again, but its values remain quite small.

As for the mixer having $\alpha=10^{\circ}$, first of all, we see that $\delta_{m}$ increases monotonically with $R e$ not only in the steady regimes but also in the unsteady asymmetric one, reaching here the maximum values. Indeed, as for T-mixers, in this regime, mixing is promoted by the presence of two co-rotating vortices in the mixing channel and the dynamic effect given by the periodic passage of the vorticity blob. At the onset of the periodic regime showing a single vortex, a drop of $\delta_{m}$ with Re occurs. However, this drop is much smaller than those observed for $\alpha=0^{\circ}$ and $20^{\circ}$, thus with values of $\delta_{m}$ remaining fairly high.

Haward et al., ${ }^{36}$ Abed et al., ${ }^{37}$ and Domingues, Poole, and Dennis $^{38}$ suggested that stagnation point flow instabilities in arrow-shaped micro-mixers and in a mixing-separating device $(\mathrm{H}$ geometry) have a supercritical behavior. Therefore, according to the previously cited papers, for $R e \geq R e_{c}$, the mixing degree should follow a square-root behavior typical of a supercritical instability. We found that the same relation holds for the T-shaped and arrow-shaped geometries investigated in the present paper in the steady engulfment regime. Mixing-degree data for $\alpha=0^{\circ}$, $10^{\circ}$, and $20^{\circ}$ are indeed well fitted by a square-root function as follows:

$$
\delta_{m}=k_{1}+k_{2} \sqrt{R e-R e_{c}},
$$

where $k_{1}$ and $k_{2}$ are two constant parameters specific for the considered flow regime and angle $\alpha$. It is interesting to note that a squareroot function well fits data also in the periodic asymmetric regime and in the periodic regime with a single vortex, when present. The fits are reported in red in Fig. 12.

To analyze the instantaneous behavior of mixing, which may be interesting, for instance, when chemical reactions take place, Fig. 14 shows the time behavior of $\delta_{m}$ at different Reynolds numbers for $\alpha=10^{\circ}$ and $20^{\circ}$. In particular, Fig. 14(a) is relative to the periodic asymmetric regime observed for $\alpha=10^{\circ}$, while Figs. 14(b) and 14(c) are relative to the periodic regime with a single vortical structure in the mixing channel for $\alpha=10^{\circ}$ and $20^{\circ}$, respectively, and Fig. 14(d) is relative to the almost periodic regime for $20^{\circ}$. The mixing degree in the asymmetric periodic regime shows significant peaks in each cycle; these peaks are due to the passage of the vorticity blob previously described. Conversely, $\delta_{m}$ is almost perfectly periodic in time in the unsteady regime with a single vortex for both $\alpha=10^{\circ}$ and $20^{\circ}$, thus confirming that mixing is mainly promoted by the periodic motion of the vortical structure in the mixing channel. Finally, in the almost periodic regime of the mixer with $\alpha=20^{\circ}$ [see Fig. 14(d)], both average $\delta_{m}$ and its variation in a cycle are much lower than in the other unsteady regimes. We observe noticeable peaks only in correspondence with the maximum tilting of the top parts of the $3 \mathrm{D}$ vortical structures (see Sec. III C). 




(a)

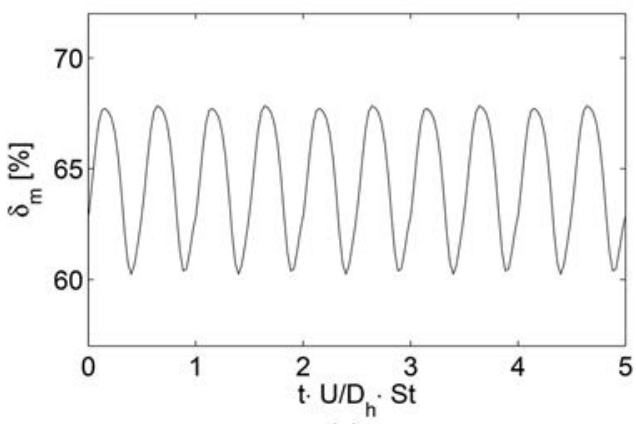

(c)

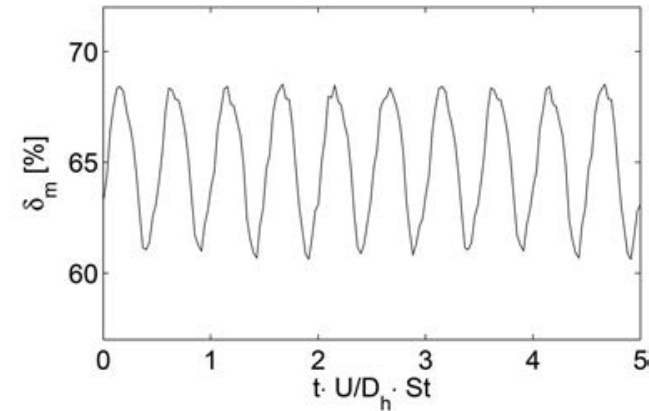

(b)

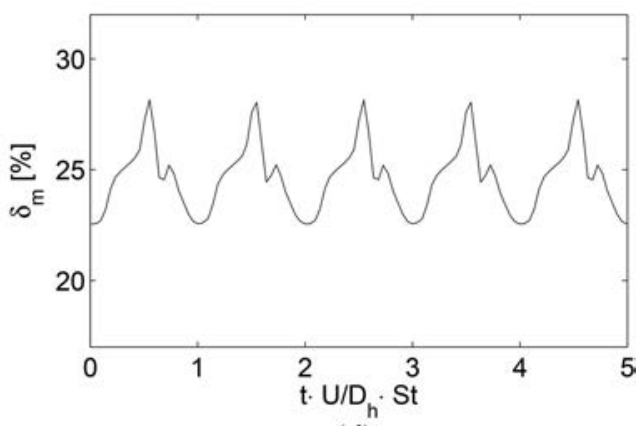

(d)

FIG. 14. Time signal of the degree of mixing evaluated at the cross section $Y=-8$ for 5 cycles: simulations carried out for (a) $R e=260$ and $\alpha=10^{\circ}$, (b) $R e=350$ and $\alpha=10^{\circ}$, (c) $R e=240$ and $\alpha=20^{\circ}$, and (d) $R e=320$ and $\alpha=20^{\circ}$.

\section{CONCLUSIONS}

The present work completes the analysis carried out in the work of Mariotti et al., ${ }^{27}$ aimed at investigating the impact of the tilting downward of the inlet channels, leading to arrow-shaped micro-mixers, on the flow topology and mixing performances. Further results, obtained in numerical simulations and supported by experimental flow visualizations, are provided and analyzed herein for higher Reynolds numbers than in the work of Mariotti et al., mainly corresponding to unsteady periodic flow dynamics. Two different tilting angles have been considered, namely, $\alpha=10^{\circ}$ and $\alpha=20^{\circ}$. We choose these values because Mariotti et al. ${ }^{27}$ showed that they present different flow behaviors and mixing performances already at lower Reynolds numbers.

We found that also at higher Reynolds numbers, the flow dynamics is significantly influenced by the tilting angle, observing different regimes for $\alpha=10^{\circ}$ and $\alpha=20^{\circ}$. In summary, for $\alpha=20^{\circ}$, the first unsteady regime, whose onset is at $R e \simeq 200$, is characterized by the periodic motion of a single vortical structure present in the mixing channel. By further increasing the Reynolds number, the flow first becomes again steady with a topology similar to that of the vortex regime occurring at much lower $R e$, and then, it becomes unsteady once more, characterized by the periodic tilting of the vortical structures near the top walls of the mixer, but by minor variations of the flow features in the mixing channel. Conversely, for the mixer with the smaller angle, $\alpha=10^{\circ}$, the first unsteady regime is practically the same as the asymmetric periodic regime occurring in T-mixers, which is characterized by the periodic shedding of a vorticity blob in the mixing channel. However, different from what happened for T-mixers, by further increasing the Reynolds number, the flow dynamics becomes very similar to that of the first regime observed for $\alpha=20^{\circ}$, i.e., the one in which a single strong vortical structure oscillates in the mixing channel.

The above differences in flow behavior have a significant impact on the mixing performances of the two arrow-shaped mixers, as analyzed in terms of the mixing degree, which was also compared to that of a T-mixer. As already shown in the work of Mariotti et al., ${ }^{27}$ the first increase in the mixing degree, corresponding to the onset of the steady engulfment regime, occurs at lower Reynolds numbers with increasing $\alpha$. However, the arrow-shaped mixer with $\alpha=20^{\circ}$ shows a non-monotonic behavior of the mixing degree with $R e$, and although in some intervals of $R e$, it has larger values than the other configurations, there are $R e$ ranges in which its mixing performances become poor. This behavior calls for careful control of the operational conditions. Conversely, the mixing degree increases monotonically with $R e$ for the arrow-mixer with $\alpha=10^{\circ}$ up to the onset of the last regime, i.e., the periodic one with a single oscillating vortex in the mixing channel, for which, however, the drop is moderate. This configuration ensures a degree of mixing, which is always larger than that of a T-mixer at Reynolds numbers above the onset of the steady engulfment. Therefore, the present study shows that a small downward tilting of the inlet channels leads to an improvement of the mixing 
performances compared to those of classical micro T-mixers, which is robust to variations in the operating conditions up to transition to a chaotic dynamics.

\section{ACKNOWLEDGMENTS}

Support by the University of Pisa through the "Progetti di Ricerca di Ateneo PRA 2017-2018" funding program is gratefully acknowledged. Computational resources from CINECA computing center (Bologna, Italy) under the ISCRA program (class B project "MIRE") are also acknowledged. Finally, the authors are grateful to Matteo Antognoli for his precious contribution to the post-processing of the numerical simulations and to Cesare Merello.

\section{DATA AVAILABILITY}

The data that support the findings of this study are available from the corresponding authors upon reasonable request.

\section{REFERENCES}

${ }^{1}$ I. Rossetti and M. Compagnoni, "Chemical reaction engineering, process design and scale-up issues at the frontier of synthesis: Flow chemistry," Chem. Eng. J. 296, 56 (2016)

${ }^{2}$ X. Yao, Y. Zhang, L. Du, J. Liu, and J. Yao, "Review of the applications of microreactors," Renewable Sustainable Energy Rev. 47, 519 (2015).

${ }^{3}$ D. M. Roberge, L. Ducry, N. Bieler, P. Cretton, and B. Zimmermann, "Microreactor technology: A revolution for the fine chemical and pharmaceutical industries?," Chem. Eng. Technol. 28, 318 (2005).

${ }^{4}$ A. Mariotti, M. Antognoli, C. Galletti, R. Mauri, M. V. Salvetti, and E. Brunazzi, "The role of flow features and chemical kinetics on the reaction yield in a T-shaped micro-reactor," Chem. Eng. J. 396, 125223 (2020).

${ }^{5}$ V. Kumar, M. Paraschivoiu, and K. D. P. Nigam, "Single-phase fluid flow and mixing in microchannels," Chem. Eng. Sci. 66, 1329 (2011).

${ }^{6}$ C.-Y. Lee, W.-T. Wang, C.-C. Liu, and L.-M. Fu, "Passive mixers in microfluidic systems: A review," Chem. Eng. J. 288, 146 (2016).

${ }^{7}$ B. Ivorra, J. López Redondo, A. M. Ramos, and J. G. Santiago, "Design sensitivity and mixing uniformity of a micro-fluidic mixer," Phys. Fluids 28, 012005 (2016).

${ }^{8}$ S. Azimi, M. Nazari, and Y. Daghighi, "Developing a fast and tunable micromixer using induced vortices around a conductive flexible link," Phys. Fluids 29, 032004 (2017)

${ }^{9}$ A. Rips and R. Mittal, "Flutter-enhanced mixing in small-scale mixers," Phys. Fluids 31, 107107 (2019).

${ }^{10}$ T. Andreussi, C. Galletti, R. Mauri, S. Camarri, and M. V. Salvetti, “Flow regimes in T-shaped micro-mixers," Comput. Chem. Eng. 76, 150 (2015).

${ }^{11}$ R. J. Poole, M. Alfateh, and A. P. Gauntlett, "Bifurcation in a T-channel junction: Effects of aspect ratio and shear-thinning," Chem. Eng. Sci. 104, 839 (2013).

${ }^{12}$ S. K. Reddy Cherlo and S. Pushpavanam, "Effect of depth on onset of engulfment in rectangular micro-channels," Chem. Eng. Sci. 65, 6486 (2010).

${ }^{13}$ A. Soleymani, H. Yousefi, and I. Turunen, "Dimensionless number for identification of flow patterns inside a T-micromixer," Chem. Eng. Sci. 63, 5291 (2008).

${ }^{14}$ C. Galletti, M. Roudgar, E. Brunazzi, and R. Mauri, "Effect of inlet conditions on the engulfment pattern in a T-shaped micro-mixer," Chem. Eng. J. 185-186, 300 (2012).

${ }^{15}$ A. Fani, S. Camarri, and M. V. Salvetti, "Investigation of the steady engulfment regime in a three-dimensional T-mixer," Phys. Fluids 25, 064102 (2013).
${ }^{16}$ N. Kockmann, T. Kiefer, M. Engler, and P. Woias, "Convective mixing and chemical reactions in microchannels with high flow rates," Sens. Actuators, B 117, 495 (2006).

${ }^{17}$ C. Galletti, G. Arcolini, E. Brunazzi, and R. Mauri, "Mixing of binary fluids with composition-dependent viscosity in a T-shaped micro-device," Chem. Eng. Sci. 123, 300 (2015).

${ }^{18}$ L. Siconolfi, S. Camarri, and M. V. Salvetti, "T-mixer operating with water at different temperatures: Simulation and stability analysis," Phys. Rev. Fluids 3, 033902 (2018).

${ }^{19}$ G. Orsi, C. Galletti, E. Brunazzi, and R. Mauri, "Mixing of two miscible liquids in T-shaped microdevices," Chem. Eng. Trans. 32, 1471 (2013).

${ }^{20}$ C. Galletti, E. Brunazzi, and R. Mauri, "Unsteady mixing of binary liquid mixtures with composition-dependent viscosity,” Chem. Eng. Sci. 164, 333 (2017).

${ }^{21}$ M. Rahimi, N. Azimi, M. A. Parsamogadam, A. Rahimi, and M. M. Masahy, "Mixing performance of T, Y, and oriented Y-micromixers with spatially arranged outlet channel: Evaluation with Villermaux/Dushman test reaction," Microsyst. Technol. 23, 3117 (2017).

${ }^{22}$ B. J. You, Y. Choi, and S. G. Im, "Influence of adjusting the inlet channel confluence angle on mixing behaviour in inertial microfluidic mixers," Microfluid. Nanofluid. 21, 121 (2017).

${ }^{23}$ H. V. Babu, S. Sahu, M. Haderlein, W. Peukert, and N. Verma, "Numerical investigation of flow patterns and concentration profiles in Y-mixers," Chem. Eng. Technol. 39, 1963 (2016).

${ }^{24}$ L. Siconolfi, A. Fani, S. Camarri, and M. V. Salvetti, "Effect of geometry modifications on the engulfment in micromixers: Numerical simulations and stability analysis," Eur. J. Mech. B: Fluids 55, 360 (2016).

${ }^{25}$ N. Kockmann and D. M. Roberge, "Transitional flow and related transport phenomena in curved microchannels," Heat Transfer Eng. 32, 595 (2011).

${ }^{26}$ C. Galletti, E. Brunazzi, L. Siconolfi, D. Spaltro, and R. Mauri, "Mixing performance of arrow-shaped micro-devices," Chem. Eng. Trans. 57, 1309 (2017).

${ }^{27}$ A. Mariotti, C. Galletti, E. Brunazzi, and M. V. Salvetti, "Steady flow regimes and mixing performance in arrow-shaped micro-mixers," Phys. Rev. Fluids 4, 034201 (2019).

${ }^{28}$ S. Dreher, N. Kockmann, and P. Woias, "Characterization of laminar transient flow regimes and mixing in T-shaped micromixers," Heat Transfer Eng. 30, 91 (2009).

${ }^{29} \mathrm{~S}$. Thomas and T. A. Ameel, "An experimental investigation of moderate Reynolds number flow in a T-channel," Exp. Fluids 49, 1231 (2010).

${ }^{30}$ A. Fani, S. Camarri, and M. V. Salvetti, "Unsteady asymmetric engulfment regime in a T-mixer," Phys. Fluids 26, 074101 (2014).

${ }^{31}$ A. Mariotti, C. Galletti, M. V. Salvetti, and E. Brunazzi, "Unsteady flow regimes in a T-shaped micromixer: Mixing and characteristic frequencies," Ind. Eng. Chem. Res. 58(29), 13340-13356 (2019).

${ }^{32}$ A. Mariotti, C. Galletti, R. Mauri, M. V. Salvetti, and E. Brunazzi, "Steady and unsteady regimes in a T-shaped micro-mixer: Synergic experimental and numerical investigation," Chem. Eng. J. 341, 414 (2018).

${ }^{33}$ P. F. Fischer, "An overlapping Schwarz method for spectral element solution of the incompressible Navier-Stokes equations," J. Comput. Phys. 133, 84 (1997).

${ }^{34}$ C. Galletti, A. Mariotti, L. Siconolfi, R. Mauri, and E. Brunazzi, "Numerical investigation of flow regimes in T-shaped micromixers: Benchmark between finite volume and spectral element methods," Can. J. Chem. Eng. 97, 528 (2019).

${ }^{35} \mathrm{~J}$. Jeong and F. Hussain, "On the identification of a vortex," J. Fluid Mech. 285, 69 (1995).

${ }^{36}$ S. J. Haward, R. J. Poole, M. A. Alves, P. J. Oliveira, N. Goldenfeld, and A. Q. Shen, "Tricritical spiral vortex instability in cross-slot flow," Phys. Rev. E 93, 031101(R)-1-031101(R)-5 (2016).

${ }^{37}$ W. M. Abed, A. F. Domingues, R. J. Poole, and D. J. C. Dennis, "Heat transfer enhancement in a cross-slot micro-geometry," Int. J. Therm. Sci. 121, 249-265 (2017).

${ }^{38}$ A. F. Domingues, R. J. Poole, and D. J. C. Dennis, "Inertial instabilities in a microfluidic mixing-separating device," Phys. Fluids 31, 074101-074116 (2019). 